\title{
Uninterruptible Power Supply Improves Precision and External Validity of Telomere Length Measurement via qPCR
}

\author{
Waylon J. Hastings ${ }^{1}$ (D), Dan T.A. Eisenberg ${ }^{2}$ (D) and Idan Shalev ${ }^{1}$ \\ ${ }^{1}$ Department of Biobehavioral Health, The Pennsylvania State University, University Park PA, USA and ${ }^{2}$ Department of \\ Anthropology, University of Washington, Seattle, WA, USA \\ Corresponding author. E-mail: whastings2012@gmail.com
}

(Received 23 September 2020; Revised 19 October 2020; Accepted 19 October 2020)

\begin{abstract}
Technical challenges associated with telomere length (TL) measurements have prompted concerns regarding their utility as a biomarker of aging. Several factors influence TL assessment via qPCR, the most common measurement method in epidemiological studies, including storage conditions and DNA extraction method. Here, we tested the impact of power supply during the qPCR assay. Momentary fluctuations in power can affect the functioning of high-performance electronics, including real-time thermocyclers. We investigated if mitigating these fluctuations by using an uninterruptible power supply (UPS) influenced TL assessment via qPCR. Samples run with a UPS had significantly lower standard deviation $(p<0.001)$ and coefficient of variation $(p<0.001)$ across technical replicates than those run without a UPS. UPS usage also improved exponential amplification efficiency at the replicate, sample, and plate levels. Together these improvements translated to increased performance across metrics of external validity including correlation with age, within-person correlation across tissues, and correlation between parents and offspring.
\end{abstract}

Keywords: telomere length; qPCR; assay precision; power supply

\section{Introduction}

Telomeres, the repetitive nucleoprotein regions at chromosome ends, are hallmarks of biological aging (Lopez-Otin et al., 2013). Large population studies have associated shorter telomere length (TL) with a range of risk factors that predict health problems and shorter life expectancy (Wang et al., 2018). Even so, technical challenges with TL measurement have led to questions regarding their utility as a biomarker of aging (e.g. Hastings et al., 2017).

The most common approach to quantify TL in epidemiological studies is quantitative-PCR (qPCR), which expresses telomeric content relative to a single copy gene (Cawthon, 2002). In addition to concerns of being less precise than measures generated by Southern Blot (Aubert et al., 2012), TL measurement via qPCR is subject to influence by several pre-analytical factors including DNA extraction method (Cunningham et al., 2013), sample storage conditions (Dagnall et al., 2017), and analytical factors such as PCR mastermix (Jiménez \& Forero, 2018) and well position on plate-based thermocyclers (Eisenberg et al., 2015). However, whether power supply during the qPCR assay influences TL measurement has remained unconsidered.

\footnotetext{
(c) The Author(s), 2020. Published by Cambridge University Press. This is an Open Access article, distributed under the terms of the Creative Commons Attribution licence (http://creativecommons.org/licenses/by/4.0/), which permits unrestricted re-use, distribution, and reproduction in any medium, provided the original work is properly cited.
} 
Momentary fluctuations in power can affect the functioning of high-performance electronics, including real-time thermocyclers. These fluctuations can be mitigated by using an uninterruptible power supply (UPS), an apparatus capable of supplying constant, uninterrupted voltage (Aamir et al., 2016). The current study investigated if UPS usage influenced TL assessment via qPCR across a range of technical and external validity metrics.

\section{Methods}

\subsection{DNA Extraction and Telomere Length Assessment}

Whole blood and buccal epithelial cells were collected from 26 grandmothers (age 52.6-72.2), 106 mothers (age 29.1-43.6) and 126 children (45.4\% male; age 0.5-24.9). DNA for TL analyses was extracted from buffy coat $(\mathrm{N}=94 ; 12$ grandmothers, 79 mothers, \& 3 children) and buccal epithelial cells $(\mathrm{N}=271 ; 26$ grandmothers, 116 mothers, \& 129 children; dataset included multiple time points for 10 mothers and 3 children) using QIAamp DNA Mini Kits (Qiagen, Germany). DNA purity and quality was assessed using 260/230 and 260/280 ratios, but no exclusionary criteria was imposed prior to assays. DNA was stored at $-80^{\circ} \mathrm{C}$ until TL analysis. All TL assays were performed by WJH on a Qiagen RotorGene Q thermocycler, using a qPCR protocol adapted from Cawthon (2002). Each telomere assay comprised two qPCR runs, one run quantifying telomere content $(\mathrm{T})$ and a second run quantifying genome copy number (S) using the single copy gene 36B4. Detailed descriptions of sample handling and processing, as well as details regarding qPCR assay and quality control are summarized in the supplemental material in accordance with guidelines recommended by the Telomere Research Network (https://rn.tulane.edu/wp-content/uploads/sites/445/2020/08/TRN-Reporting-Guidelines-updated.pdf). The same DNA aliquot was used for T and S runs. Each run hosted triplicate reactions of 22 samples, 5 standards, and 6 positive controls on 100 well disks.

Telomere length was quantified as the T/S ratio, which was calculated as $T / S=\left(\frac{E_{T}{ }^{c} t_{T}}{E_{S}}\right)^{-1}$, where $\mathrm{E}_{\mathrm{T} / \mathrm{S}}$ is the efficiency of exponential amplification for the telomere or single copy gene respectively, and $\mathrm{Ct}_{\mathrm{T} / \mathrm{S}}$ is the cycle at which a given replicate targeting telomeric content or the single copy gene reaches the critical threshold of fluorescence detection. A serial dilution of five standards were used to identify a critical threshold of detection for extraction of $\mathrm{Ct}_{\mathrm{T} / \mathrm{S}}$ values. Estimates of amplification efficiency at the replicate, sample, and plate levels used data generated from LinRegPCR (Ramakers et al., 2003). T/S ratios were calculated using plate-level efficiencies, which have been shown to decrease bias and variability in qPCR data (Ruijter et al., 2009).

\subsection{Sample Overview and Statistical Analyses}

The present work summarizes data generated from TL assessments of 2,221 replicate reactions across $34 \mathrm{qPCR}$ runs (17 T \& $17 \mathrm{~S}$ ) as part of a larger investigation into intergenerational transmission of trauma, as previously reported (Etzel et al., 2020). Sample-level analyses used the standard deviation and coefficient of variation across replicate $\mathrm{Ct}_{\mathrm{T} / \mathrm{S}}$ values, natural $\log$ transformed $\mathrm{T}$ estimates $\left(\operatorname{Ln}\left[E_{T}{ }^{C t_{T}}\right]\right)$, and natural $\log$ transformed $S$ estimates $\left(\operatorname{Ln}\left[E_{S}{ }^{C t_{S}}\right]\right.$ ). Due to potential differences in reaction chemistry, telomere and single copy gene reactions were analyzed independently. A full break down of sample flow and subsets used in each analysis is provided in Figure 1. Results described in the main text represent combined analyses of leukocyte and buccal samples. Independent analyses within each tissue are reported in Supplemental Tables S1-S5. Two telomere samples failing to reach the threshold of detection were removed from analyses. A UPS (Back-UPS Pro 700; APC) was utilized on approximately half of the runs $(\mathrm{N}=18 ; 9 \mathrm{~T} \& 9 \mathrm{~S})$. All samples which had $\mathrm{T}$ run with the UPS also had their corresponding $\mathrm{S}$ reaction also run with the UPS. The runs utilizing the UPS were situated within the middle of the assays (i.e., $5 \mathrm{~T}$ runs and $5 \mathrm{~S}$ runs without the UPS followed by $9 \mathrm{~T}$ runs and $9 \mathrm{~S}$ runs using the UPS followed by 4 $\mathrm{T}$ runs and $4 \mathrm{~S}$ runs without the UPS). Differences in group means were assessed using t-tests. Homogeneity of variances between reactions assessed with and without the UPS was tested using 


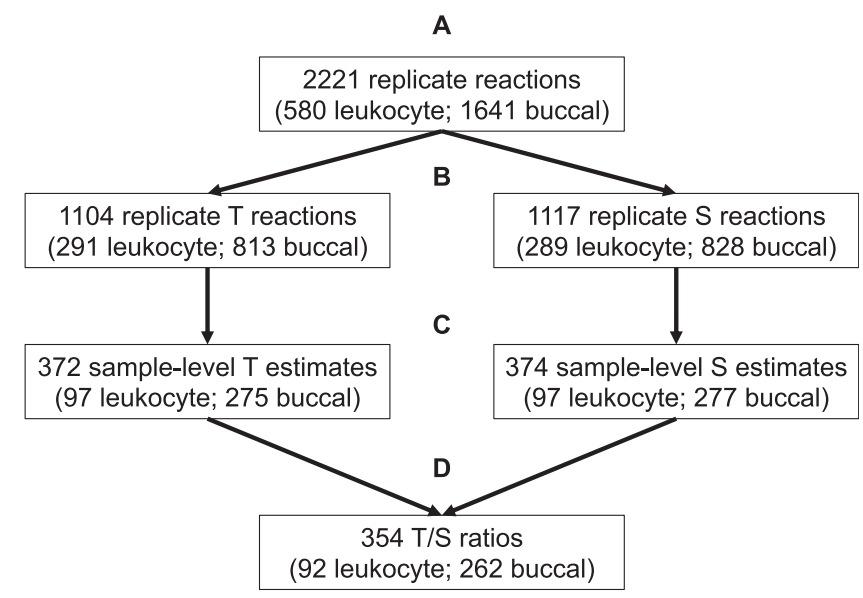

Figure 1. Sample flow and subsets used for analyses. 1A. 2,221 replicate reactions comprising the full sample. 1B. Replicate reactions were distinguished by amplification target and analyzed separately due to concerns in reaction chemistry. Replicate reactions were used in analyses of replicate level efficiencies as a function of UPS utilization. 1C. Technical replicates were clustered by sample ID for analyses of standard deviation and coefficient of variation across replicate level efficiencies, replicate T-estimates, and replicate S-estimates. Differences in sample level efficiencies, calculated as the average efficiency across replicates, were also conducted within this subsample. The two additional data points for single copy gene data correspond to the two telomere samples that did not amplify as described in main text. 1D. Calculated T-estimates and S-estimates were used to calculate T/S values for 363 samples. Original T/S values for the 9 samples that were rerun were not included in analyses of T/S ratio data. Neither were the $9 \mathrm{~T} / \mathrm{S}$ values marked as outliers, bringing the final sample size for external validity correlates to 354 .

Levene's test. In instances where group variances were significantly different, the Welch $t$ test was conducted in lieu of the Student's t test (Welch, 1947).

To better understand how UPS usage would influence the findings derived from telomere length data, we compared how samples assessed on runs with and without a UPS varied in their correlation between $\mathrm{T} / \mathrm{S}$ ratio and external validity metrics including age, across-tissue within person, and among parents and offspring (Eisenberg, 2016). Differences in T/S ratio correlation coefficients were evaluated based on overlap of $83.4 \%$ confidence intervals (Knol et al., 2011). Samples with T/S ratios more than 3 standard deviations above the mean were marked as outliers and removed from analyses $\left(\mathrm{N}_{(+) \text {UPS }}=5 ; \mathrm{N}_{(-) \text {UPS }}=4\right)$. Statistical analyses were conducted with IBM SPSS Statistics 26 . Sample size estimates for reported power calculations were performed in Stata 15.1 using the 'power onecorrelation' command with power $=0.80$ and $\alpha=0.05$, and effect size equal to the observed correlation coefficient.

\section{Results}

The standard deviation and coefficient of variation were significantly lower across replicate $\mathrm{Ct}_{\mathrm{T}}$ values and natural $\log$ transformed $\mathrm{T}$ estimates for samples assessed on runs utilizing a UPS relative to those run without a UPS (Figure 2A;Table 1:). Estimates of amplification efficiency at the replicate, sample, and plate level were also significantly improved on runs using a UPS, situating them closer to desired population doubling (Figure 3A; Table 1:). Similar patterns were observed for reactions targeting the single copy gene (Figure 2B; Figure 3B; Table 2:).

UPS status improved all metrics assessing the external validity of T/S ratios (Table 3:). Within-person, cross-tissue correlations were significantly higher for samples assessed with a UPS relative to those without. The correlation between age and T/S ratios and between parent and offspring T/S ratios were also improved for samples assessed with a UPS, but not to a significant extent. Similar patterns were observed when leukocyte and buccal samples were analyzed independently (Supplemental Table S5).

To explore power gains yielded from using the UPS, we compared the sample sizes needed to distinguish UPS versus no-UPS TL external validity correlates as significantly different from zero 
A

A Impact of UPS on qPCR Precision (T Reactions)

$\square(+)$ UPS $\square(-)$ UPS

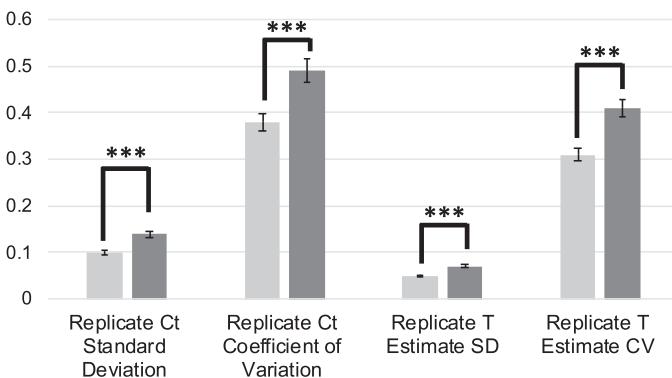

B

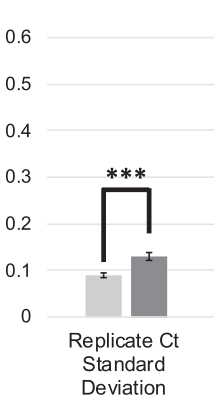

$\square(+)$ UPS $\square(-)$ UPS

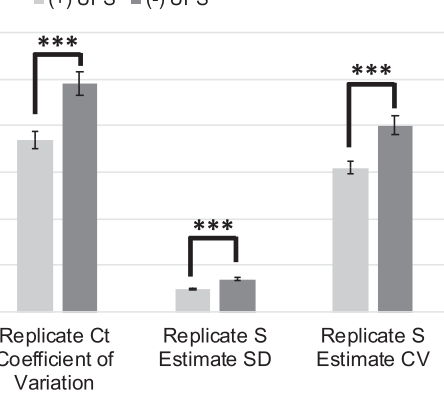

Figure 2. Differences in assay precision as a function of UPS usage delineated by PCR amplification target. 2A: Average standard deviation and coefficient of variation across replicate $\mathrm{Ct}_{\mathrm{T}}$ values and $\mathrm{T}$ estimates for samples assessed with $(\mathrm{N}=196)$ and without ( $\mathrm{N}=176)$ the use of a UPS. 2B: Average standard deviation and coefficient of variation across replicate $\mathrm{Ct}_{\mathrm{S}}$ values and $\mathrm{S}$ estimates for samples assessed with $(\mathrm{N}=198)$ and without $(\mathrm{N}=176)$ the use of a UPS. Error bars reflect standard error of the mean. $\mathrm{SD}=$ Standard Deviation. $\mathrm{CV}=\mathrm{Coefficient}$ of Variation. ${ }^{* \star *} p<0.001$.

Table 1. Comparing Features of T-Estimates by UPS Status

\begin{tabular}{lcccc}
\hline & $(-$ UPS $)$ & $(+)$ UPS & Test Statistic & $p$-value \\
\hline Standard Deviation Across Replicate Ct Values & $0.14(0.09)$ & $0.10(0.07)$ & $t_{322.038}=4.555$ & $p<0.001$ \\
\hline CV Across Replicate Ct Values & $0.49(0.32)$ & $0.38(0.25)$ & $t_{330.442}=3.829$ & $p<0.001$ \\
\hline $\begin{array}{l}\text { Standard Deviation Across Replicate Natural Log } \\
\quad \text { Transformed T-Estimates }\end{array}$ & $0.07(0.04)$ & $0.05(0.03)$ & $t_{329.618}=4.294$ & $p<0.001$ \\
\hline CV Across Replicate Natural Log Transformed T-Estimates & $0.41(0.26)$ & $0.31(0.20)$ & $t_{331.142}=3.909$ & $p<0.001$ \\
\hline Replicate Level Efficiency & $1.88(0.08)$ & $1.89(0.05)$ & $t_{921.119}=-3.571$ & $p<0.001$ \\
\hline Standard Deviation Across Replicate Level Efficiencies & $0.05(0.03)$ & $0.04(0.02)$ & $t_{293.912}=5.979$ & $p<0.001$ \\
\hline Coefficient of Variation Across Replicate Level Efficiencies & $2.79(1.51)$ & $1.88(0.97)$ & $t_{292.945}=6.131$ & $p<0.001$ \\
\hline Sample Level Efficiency & $1.88(0.05)$ & $1.89(0.04)$ & $t_{311.781}=-2.963$ & $p=0.003$ \\
\hline Plate Level Efficiency & $1.87(0.03)$ & $1.89(0.02)$ & $t_{15}=-2.430$ & $p=0.028$ \\
\hline
\end{tabular}

${ }^{*}$ Test statistics reported from independent samples t-test. Values reported are Mean (Standard Deviation). CV=coefficient of variation.

A Impact of UPS on Amplification Efficiency (T Reactions)

$=(+)$ UPS $=(-)$ UPS

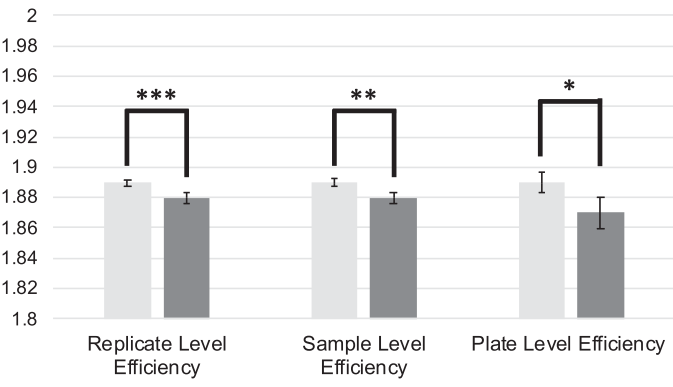

B

Impact of UPS on Amplification Efficiency (S Reactions)

$=(+)$ UPS $=(-)$ UPS

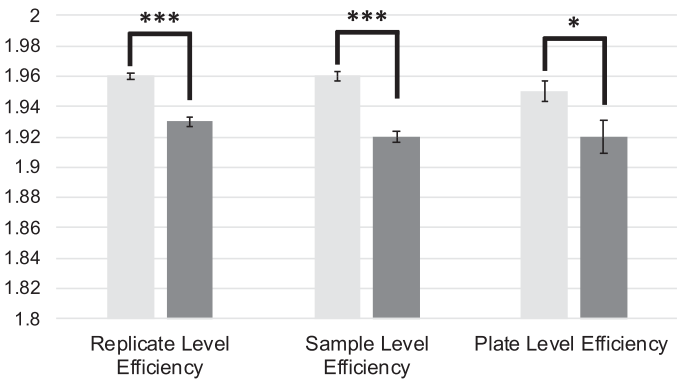

Figure 3. Differences in amplification efficiency as a function of UPS usage delineated by PCR amplification target. 3A: Average replicate, sample, and plate-level efficiencies for telomere reactions assessed with and without the use of a UPS. 3B: Average replicate, sample, and plate-level efficiencies for single copy gene reactions assessed with and without the use of a UPS. Efficiencies derived from LinRegPCR. Error bars reflect standard error of the mean. SD = Standard Deviation. CV=Coefficient of Variation. ${ }^{\star \star \star} p<0.001 ;{ }^{\star \star} p<0.01 ;{ }^{\star} p<0.05$. 
Table 2. Comparing Features of S-Estimates by UPS Status

\begin{tabular}{lcccc}
\hline & $(-$ UPS $)$ & $(+)$ UPS & Test Statistic & $p$-value \\
\hline Standard Deviation Across Replicate Ct Values & $0.13(0.09)$ & $0.09(0.06)$ & $t_{321.314}=4.571$ & $p<0.001$ \\
\hline CV Across Replicate Ct Values & $0.49(0.33)$ & $0.37(0.26)$ & $t_{332.150}=3.804$ & $p<0.001$ \\
\hline $\begin{array}{l}\text { Standard Deviation Across Replicate Natural Log } \\
\quad \text { Transformed S-Estimates }\end{array}$ & $0.07(0.05)$ & $0.05(0.03)$ & $t_{324.946}=4.267$ & $p<0.001$ \\
\hline CV Across Replicate Natural Log Transformed S-Estimates & $0.40(0.27)$ & $0.31(0.21)$ & $t_{332.144}=3.818$ & $p<0.001$ \\
\hline Replicate Level Efficiency & $1.93(0.08)$ & $1.96(0.06)$ & $t_{976.353}=-7.431$ & $p<0.001$ \\
\hline Standard Deviation Across Replicate Level Efficiencies & $0.05(0.03)$ & $0.04(0.02)$ & $t_{318.043}=4.672$ & $p<0.001$ \\
\hline Coefficient of Variation Across Replicate Level Efficiencies & $2.57(1.37)$ & $1.94(1.01)$ & $t_{318.327}=4.955$ & $p<0.001$ \\
\hline Sample Level Efficiency & $1.92(0.05)$ & $1.96(0.04)$ & $t_{328.426}=-6.365$ & $p<0.001$ \\
\hline Plate Level Efficiency & $1.92(0.03)$ & $1.95(0.02)$ & $t_{15}=-2.841$ & $p=0.012$ \\
\hline
\end{tabular}

${ }^{*}$ Test statistics reported from independent samples t-test. Values reported are Mean (Standard Deviation). CV=coefficient of variation.

Table 3. Comparing Metrics of External Validity by UPS Status

\begin{tabular}{|c|c|c|}
\hline \multicolumn{3}{|c|}{ Leukocyte-Buccal Correlation of Plate-Level T/S Ratios } \\
\hline & $r$ (p-value) & $83.4 \% \mathrm{Cl}$ \\
\hline (-) UPS & $0.62(<0.001)$ & {$[0.47,0.74]$} \\
\hline (+) UPS & $0.92(<0.001)$ & {$[0.88,0.95]$} \\
\hline \multicolumn{3}{|c|}{${ }^{\star}$ Correlations controlled for sex and age } \\
\hline \multicolumn{3}{|c|}{ Correlation Between Age and Plate-Level T/S Ratios } \\
\hline & $r$ (p-value) & $83.4 \% \mathrm{Cl}$ \\
\hline$(-)$ UPS & $-0.13(0.085)$ & {$[-0.23,-0.02]$} \\
\hline$(+)$ UPS & $-0.15(0.048)$ & {$[-0.26,-0.05]$} \\
\hline \multicolumn{3}{|c|}{${ }^{\star}$ Correlations controlled sex and tissue (leukocyte/buccal) } \\
\hline \multicolumn{3}{|c|}{ Parent-Offspring Correlation of Plate-Level T/S Ratios } \\
\hline & $r$ ( $p$-value) & $83.4 \% \mathrm{Cl}$ \\
\hline$(-)$ UPS & $0.74(<0.001)$ & {$[0.65,0.80]$} \\
\hline (+) UPS & $0.78(<0.001)$ & {$[0.70,0.84]$} \\
\hline
\end{tabular}

* Correlations controlled for sex, parental age, offspring age, and tissue (leukocyte/buccal)

$(\alpha=0.05$, power $=0.80$.) For example, to detect the correlation of TL across tissues without a UPS $(r=$ $0.62)$ requires a sample size of 18 , while a sample of 7 is required to detect to detect with a UPS $(r=0.92)$. This equates to being able to detect a significant effect with a $61 \%$ smaller sample size. Using the same procedure for age and parent-offspring correlations yields estimates of $25 \%$ and $8 \%$ smaller samples, for an average ability to detect an effect with a $31 \%$ smaller sample.

\section{Conclusions}

TL assessment via qPCR is subject to bias from a host of analytical and pre-analytical factors (reviewed in Lin et al., 2019), leading some to challenge the utility of telomeres as a biomarker of aging (Boonekamp 
et al., 2013). Nevertheless, TL measurement via qPCR remains widely used in epidemiological research. Thus, elucidating measurement practices which enhance reproducibility and precision is of great interest.

Our results demonstrate substantial improvements to qPCR assay precision and measures of external validity through the utilization of an uninterruptible power supply. Further, findings suggest utilization of a UPS increases power in a manner equivalent to a $31 \%$ increase in sample size, although the degree of such improvement may differ with the baseline electric power quality and type of thermocycler employed. We frame our findings in the context of literature on TL assessment given the aim of the assays comprising our sample. However, the results are likely applicable to qPCR more broadly, and demonstrate the importance of considering power supply when conducting biological assays that rely on high performance electronics.

Acknowledgements. The authors would like to thank Brooke C. Mattern for their assistance with DNA extraction and quantification. We would also like to thank Dr Sue Rutherford Siegel in the Department of Biobehavioral Health Biomarker Core Lab for allowing the use of the facilities to perform telomere length assays.

Author Contributions. WJH and IS conceived original study that was refined by DTAE. WJH conducted telomere length assays. DTAE conceived analytical approach. WJH performed statistical analyses. DTAE performed power calculations. WJH, IS, and DTAE wrote the article.

Funding Information. This work was supported by National Institute on Aging (WJH, grant number T32AG049676) and National Institute of Environmental Health Sciences (IS grant number U01ES030949). The content is solely the responsibility of the authors and does not necessarily represent the official views of the National Institutes of Health.

Conflict of Interest. WJH, DTAE, and IS declare none.

Data Availability. The dataset used to generate the presented findings is available for public download at the repository site figshare (doi: 10.6084/m9.figshare.12954899).

Supplementary Materials. To view supplementary material for this article, please visit http://dx.doi.org/10.1017/exp.2020.58.

\section{References}

Aamir, M., Kalwar, K. A., \& Mekhilef, S. (2016). Review: Uninterruptible power supply (UPS) system. Renewable and Sustainable Energy Reviews, 58, 1395-1410. https://doi.org/10.1016/j.rser.2015.12.335.

Aubert, G., Hills, M., \& Lansdorp, P. M. (2012). Telomere length measurement-caveats and a critical assessment of the available technologies and tools. Mutation Research, 730, 59-67. https://doi.org/10.1016/j.mrfmmm.2011.04.003.

Boonekamp, J. J., Simons, M. J. P., Hemerik, L., \& Verhulst, S. (2013). Telomere length behaves as biomarker of somatic redundancy rather than biological age. Aging Cell, 12, 330-332. https://doi.org/10.1111/acel.12050.

Cawthon, R. M. (2002). Telomere measurement by quantitative PCR. Nucleic Acids Research, 30, e47. http://www.ncbi.nlm.nih. gov/pubmed/12000852.

Cunningham, J. M., Johnson, R. A., Litzelman, K., Skinner, H. G., Seo, S., Engelman, C. D., Vanderboom, R. J., Kimmel, G. W., Gangnon, R. E., Riegert-Johnson, D. L., Baron, J. A., Potter, J. D., Haile, R., Buchanan, D. D., Jenkins, M. A., Rider, D. N., Thibodeau, S. N., Petersen, G. M., \& Boardman, L. A. (2013). Telomere length varies by DNA extraction method: Implications for epidemiologic research. Cancer Epidemiology, Biomarkers \& Prevention, 22, 2047-2054. https:// doi.org/10.1158/1055-9965.EPI-13-0409.

Dagnall, C. L., Hicks, B., Teshome, K., Hutchinson, A. A., Gadalla, S. M., Khincha, P. P., Yeager, M., \& Savage, S. A. (2017) Effect of pre-analytic variables on the reproducibility of qPCR relative telomere length measurement. PLoS ONE, 12, e0184098. https://doi.org/10.1371/journal.pone.0184098.

Eisenberg, D. T. A. (2016). Telomere length measurement validity: The coefficient of variation is invalid and cannot be used to compare quantitative polymerase chain reaction and Southern blot telomere length measurement techniques. International Journal of Epidemiology, 45, 1295-1298. https://doi.org/10.1093/ije/dyw191.

Eisenberg, D. T. A., Kuzawa, C. W., \& Hayes, M. G. (2015). Improving qPCR telomere length assays: Controlling for well position effects increases statistical power. American Journal of Human Biology, 27, 570-575. https://doi.org/10.1002/ ajhb.22690.

Etzel, L., Hastings, W. J., Mattern, B. C., Oxford, M. L., Heim, C., Putnam, F. W., Noll, J. G., \& Shalev, I. (2020). Intergenerational transmission of childhood trauma? Testing cellular aging in mothers exposed to sexual abuse and their children. Psychoneuroendocrinology, 120, 104781. https://doi.org/10.1016/j.psyneuen.2020.104781. 
Hastings, W. J., Shalev, I., \& Belsky, D. W. (2017). Translating measures of biological aging to test effectiveness of geroprotective interventions: What can we learn from research on telomeres?. Frontiers in Genetics, 8, 164. https://doi. org/10.3389/fgene.2017.00164.

Jiménez, K. M., \& Forero, D. A. (2018). Effect of master mixes on the measurement of telomere length by qPCR. Molecular Biology Reports, 45, 633-638. https://doi.org/10.1007/s11033-018-4175-y.

Knol, M. J., Pestman, W. R., \& Grobbee, D. E. (2011). The (mis)use of overlap of confidence intervals to assess effect modification. European Journal of Epidemiology, 26, 253-254. https://doi.org/10.1007/s10654-011-9563-8.

Lin, J., Smith, D. L., Esteves, K., \& Drury, S. (2019). Telomere length measurement by qPCR - Summary of critical factors and recommendations for assay design. Psychoneuroendocrinology, 99, 271-278. https://doi.org/10.1016/j.psyneuen.2018.10.005.

Lopez-Otin, C., Blasco, M. A., Partridge, L., Serrano, M., \& Kroemer, G. (2013). The hallmarks of aging. Cell, 153, $1194-1217$. https://doi.org/10.1016/j.cell.2013.05.039.

Ramakers, C., Ruijter, J. M., Deprez, R. H. L., \& Moorman, A. F. M. (2003). Assumption-free analysis of quantitative realtime polymerase chain reaction (PCR) data. Neuroscience Letters, 339, 62-66. https://doi.org/10.1016/S0304-3940(02)014234.

Ruijter, J. M., Ramakers, C., Hoogaars, W. M. H., Karlen, Y., Bakker, O., van den Hoff, M. J. B., \& Moorman, A. F. M. (2009). Amplification efficiency: Linking baseline and bias in the analysis of quantitative PCR data. Nucleic Acids Research, 37, e45. https://doi.org/10.1093/nar/gkp045.

Wang, Q., Zhan, Y., Pedersen, N. L., Fang, F., \& Hägg, S. (2018). Telomere length and all-cause mortality: A meta-analysis. Ageing Research Reviews, 48, 11-20. https://doi.org/10.1016/j.arr.2018.09.002.

Welch, B. L. (1947). The generalization of 'student's' problem when several different population variances are involved. Biometrika, 34, 28-35. https://doi.org/10.2307/2332510.

Cite this article: Hastings WJ, Eisenberg DTA, Shalev I (2020). Uninterruptible Power Supply Improves Precision and External Validity of Telomere Length Measurement via qPCR Experimental Results, 1, e52, 1-11. https://doi.org/ $10.1017 /$ exp. 2020.58 


\section{Peer Reviews}

\section{Reviewing editor: Dr. Michael Nevels}

University of St Andrews, Biomolecular Sciences Building, Fife, United Kingdom of Great Britain and Northern Ireland, KY16 9ST

This article has been accepted because it is deemed to be scientifically sound, has the correct controls, has appropriate methodology and is statistically valid, and has been sent for additional statistical evaluation and met required revisions.

doi:10.1017/exp.2020.58.pr1

\section{Review 1: Uninterruptible Power Supply Improves Precision and External Validity of Telomere Length Measurement via qPCR}

Reviewer: Dr. Francisco Morinha

Date of review: 07 October 2020

(C) The Author(s), 2020. Published by Cambridge University Press This is an Open Access article, distributed under the terms of the Creative Commons Attribution licence (http://creativecommons.org/licenses/by/4.0/), which permits unrestricted re-use, distribution, and reproduction in any medium, provided the original work is properly cited.

Conflict of interest statement. Reviewer declares none.

Comments to the Author: The work is very interesting and a good contribution for telomere biologists working with qPCR measurements in different research fields, and may have some repercussions for those who use qPCR for other applications.

I have only some remarks:

1. Sampling description needs to be improved.

- How many samples of buffy coat and buccal epithelial cells were collected from each group (grandmothers, mothers and children)?

- How DNA quality/purity was assessed? Please describe the technique and the sample exclusion criteria.

- DNA was extracted from buffy coat $(\mathrm{N}=94)$ and buccal epithelial cells $(\mathrm{N}=269)$. Did these DNA samples all have good quality for measuring telomeres by qPCR?

2. How many days/weeks did the assays take? Please indicate the precise time period (days/weeks or months) of assays with and without UPS.

3. How was the DNA stored? The working solution was always the same DNA aliquot? I think that the freezethaw cycles of the samples varied between assays. This can be a very critical point for qPCR telomere assays. It is important to indicate how this factor was controlled.

4. Were the assays always performed by the same person? Please state in the manuscript.

5. Lines 69-70: Each sample was analyzed in duplicate, triplicate...? Please describe in the manuscript. How many samples were analyzed per run/plate? Saying 34 PCR runs is unclear.

6. Line 59: It would be interesting for the reader to see at least the TL primers and the single-copy gene name and primers used in this work. 
Is the data presented in the most useful manner? (40\%) 4/5

Does the paper cite relevant and related articles appropriately? (30\%) 4/5

Context

Does the title suitably represent the article? (25\%)

Does the abstract correctly embody the content of the article? (25\%)

Does the introduction give appropriate context? (25\%)

Is the objective of the experiment clearly defined? (25\%)

Analysis

Are the limitations of the experiment as well as the contributions of the experiment clearly outlined? $(20 \%)$ 


\title{
Review 2: Uninterruptible Power Supply Improves Precision and External Validity of Telomere Length Measurement via qPCR
}

\author{
Reviewer: Dr. Stacy Drury (D) \\ Tulane University, Louisiana, United States \\ Date of review: 13 October 2020
}

\begin{abstract}
(C) The Author(s), 2020. Published by Cambridge University Press This is an Open Access article, distributed under the terms of the Creative Commons Attribution licence (http://creativecommons.org/licenses/by/4.0/), which permits unrestricted re-use, distribution, and reproduction in any medium, provided the original work is properly cited.
\end{abstract}

Conflict of interest statement. reviewer declares none

Comments to the Author: This is an important methodologic manuscript. Please address (1) sample flow and what subsets were included in each analysis, include a flow chart (2) assay methodology using the TRN reporting guidelines for qPCR based TL studies and include an enhanced description of the 744 replicates (e.g. sample? plate?) (3) use ICCs instead of CV's. There were 34 runs, $17 \mathrm{~T}$ and $17 \mathrm{~S}$, but 18 used the UPS as such comparisons with the T/S ratio for any analyses would include samples that had one plate with the T or S on the UPS and one without the UPS, please clarify and justify if appropriate. The analytic section needs greater details and should match the language and analyses in the results. Please define "external validity correlations" in methods/analytic section. Please better define "homogeneity of variance" and what aspects of the study this refers to as well as the analytic method to characterize it. The results refer to a "subset" however it is unclear what subset this refers to. Samples with a T/S ratio > 3sd were removed, however the analytic approach considered T and S plates separately, please provide justification for this approach. Please describe the analytic approach for the power analyses in the methods. There remains a debate about a true "gold standard" for TL analyses, please remove this statement. Placing this study in the broader qPCR field and determining if the UPS have been evaluated for other qPCR applications would broaden the applicability of the study results.

\section{Score Card}

Presentation

3.9

Is the article written in clear and proper English? (30\%)

Is the data presented in the most useful manner? (40\%)

Does the paper cite relevant and related articles appropriately? (30\%)

Context

Does the title suitably represent the article? (25\%)

Does the abstract correctly embody the content of the article? (25\%)

Does the introduction give appropriate context? (25\%)

Is the objective of the experiment clearly defined? (25\%) 
Are the limitations of the experiment as well as the contributions of the experiment clearly outlined? (20\%) 